PAPERS

\title{
Average temperature of oil-filled transformer windings with partial cooling ducts
}

\author{
Peter Bokes
}

\begin{abstract}
An effective one-dimensional model is presented that describes the temperature profile of a winding of an oil-filled distribution transformer with an arbitrary number of partial cooling ducts. An analytical solution of the model is applied to a specific example - a low voltage winding of a $400 \mathrm{kVA}$ distribution transformer with one or two partial cooling ducts. Starting from the exact solution, a simple and practical formula for the temperature rise of similar windings has been derived that is suitable for transformer designers.
\end{abstract}

K e y w or d s: : heat transfer, distribution transformers, temperature rise, partial cooling ducts

\section{Introduction}

Practising designers of power and distribution transformers like to say that transformer design is one of the most conservative areas of engineering. Indeed, the three basic components - magnetic core, windings and the casing, at first glance, have changed little over the last 80 years. Nevertheless, the demand for more reliable transformers, economical both in service and in production, together with an increase of the prices of input commodities, has resulted in many ingenious innovations [1]. Among them are also innovations in the design of cooling ducts or channels in the windings of oil-filled transformers. Cooling ducts direct mineral oil through the windings and thereby transfer Joule losses away. For a producer it is necessary to guarantee that the temperature rise of the winding fulfils the limits set by international standards [2]. However, making cooling ducts too generous increases the size of the winding and magnetic core which both lead to unnecessary increase of the needed material and hence the price. Traditionally (ie before 1990 [3]) cooling ducts extended along the whole circumference of the winding. In this case its average temperature is given by the wellknown expression [4]

$$
\vartheta_{\mathrm{av}}=\vartheta_{\mathrm{oil}}+\frac{\dot{q} h}{2 \alpha}+\frac{\dot{q} h^{2}}{12 \lambda_{\perp}}
$$

where $\vartheta_{\text {oil }}$ is the average temperature of the oil, $\dot{q}$ is the volume density of Joule losses, $h$ is the radial width of the winding, $\alpha$ is the heat transfer coefficient between the winding's surface and oil in the duct and $\lambda_{\perp}$ is the thermal conductivity of the winding in its radial direction. The second contribution in (1) is the increase of the temperature within the oil's boundary layer. It is well-known [4] that this part of the heat transfer is nonlinear so one should view this contribution as a linear approximation close to typical temperatures of the winding. This turns out to be satisfactory for our discussion as well as the practical formulae given in the next sections. Finally, the third contribution gives the temperature rise in the solid part.

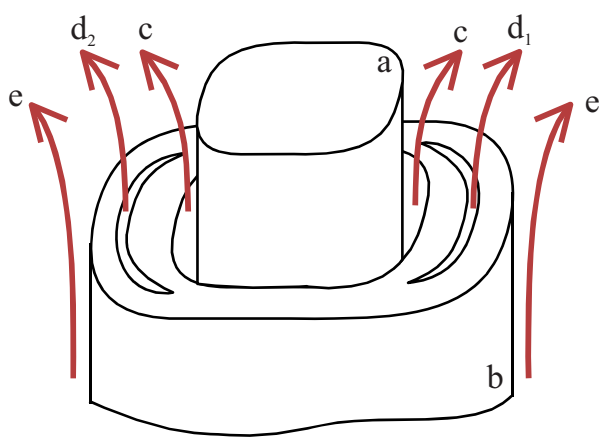

Fig. 1. A sketch of the upper part of one leg of a three-phase transformer

The innovation of the cooling ducts design for distribution transformers that addressed the contradicting demands of their heat transfer capacity and their size was to use partial cooling ducts, Fig. 1. Here, the cooling ducts extend only along a fraction of the overall circumference of the winding. In figure, (a) - is the oval-shaped magnetic core, and (b) - is the low voltage winding wound around. Mineral oil flows along its surface (c),(e) as well as through the partial cooling ducts $\left(d_{1}\right)$ and $\left(d_{2}\right)$ that do not extend around the whole circumference.

The partial cooling ducts are placed into that part of the winding that extrudes outside from the yoke of the magnetic circuit. In principle, the designer may choose to employ only one partial cooling duct $\left(e g \mathrm{~d}_{1}\right.$ in Fig. 1$)$ if

Slovak University of Technology in Bratislava, Faculty of Electrical Engineering and Information Technology, Institute of Nuclear and Physical Engineering, Bratislava 812 19, Slovakia, peter.bokes@stuba.sk 
it is enough to keep the temperature rise below the limiting value. The merit of using the partial cooling ducts is the decrease of the amount of electrical steel needed for the upper and lower yoke of the iron core as well as the amount of the wire. Interestingly, the concept of partial cooling ducts has recently been given a new twist for transformers with circular cross-section of magnetic cores [5].

On the other hand, the complication that partial ducts introduced for the designer was the loss of the approximate cylindrical symmetry of the problem which raises questions about the use of the traditional expression for the calculation of the average temperature (1). The temperature now depends on the selected position along the winding in its tangent direction and its average temperature must be obtained by some specific average procedure. In this paper both questions are answered for a general case of winding with arbitrary number of partial cooling ducts. A simple but perhaps most frequent case of a single cooling duct on one or both sides of the winding is analysed in greater detail and a simplified formula for the temperature rise is obtained.

\section{Averaging procedure for an asymmetric winding}

Average temperature of winding is measured indirectly through the measurement of the resistance of windings $R$. Since the temperature along the wire is not constant, we have to calculate it by adding infinitesimal resistances of the wound wire that depend on their local temperature,

$$
R=\int_{0}^{L} \frac{\mathrm{d} R}{\mathrm{~d} l} \mathrm{~d} l=R_{0}\left[1+\beta\left(\frac{1}{L} \int_{0}^{L} \vartheta(l) \mathrm{d} l-\vartheta_{0}\right)\right]
$$

where $L$ is the length of the wire, $R_{0}$ the resistance of the wire at reference temperature $\vartheta_{0}$ and $\beta$ is the temperature coefficient of the resistance. Therefore, the average temperature of the wire $\vartheta_{\mathrm{av}}$ is defined by the integral along the wire,

$$
\vartheta_{\mathrm{av}}=\frac{1}{L} \int_{0}^{L} \vartheta(l) \mathrm{d} l
$$

\section{Mathematical model and its solution}

A cross-sectional view of a winding with partial cooling ducts on both sides is shown in Fig. 2. Due to the symmetry of the problem only one quarter of the winding has to be considered in the model. Within the first quadrant the effective one-dimensional model describes the variation of the temperature around the circumference of the winding in three different segments $i=0,1$ and 2 , all having the origin of their respective coordinates $x_{i} \in\left(0, l_{i}\right)$ in the plane $O$. The widths of the segments $h_{\mathrm{i}}$ are indicated as well.
The straight segment of length $l_{0}$ and width $h_{0}$ is split by the partial cooling duct into two segments of lengths $l_{1}$ and $l_{2}$ and widths $h_{1}$ and $h_{2}$ respectively at the position of the plane $O$. Within each segment the temperature changes along both radial and tangential directions. Since we are primarily interested in the latter, we will calculate temperature profile $\vartheta_{i}(x)$ that is averaged over the radial direction and depends on the tangential coordinate $x$ only. Our model also assumes that the dependence of the temperature along the axial direction of the winding (perpendicular to the cross-section in Fig. 2) is unimportant and the actual calculated quantities give the average along the axial height of the winding $l_{w}$.

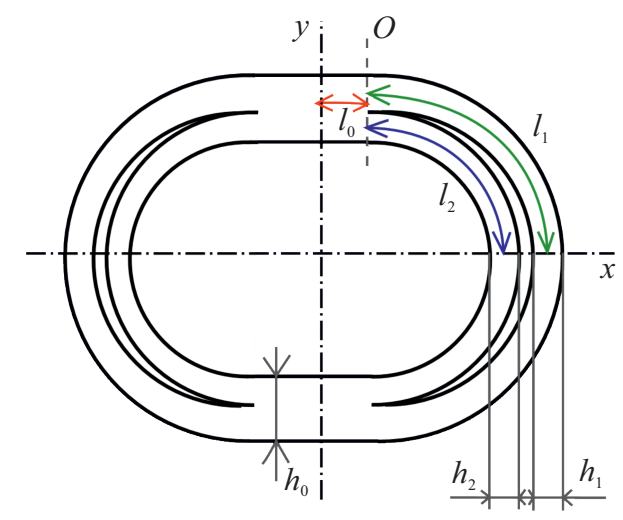

Fig. 2. Cross-sectional view of the winding from Fig. 1

It is straightforward to generalize the situation with one segment split into two for a case of one segment split into $N-1$ segments separated by $N-2$ cooling ducts. We will use an index $i$ for the coordinate $x$ whenever it is important to distinguish its values between different segments. For example, within each segment the coordinates run from $x_{i}=0$, set in the plane $O$, up to the length of the corresponding segment $x_{i}=l_{i}$. The average temperature (3) can be calculated from the temperature profiles within each segment $\vartheta_{i}(x)$. There are $n_{i}$ turns of wire of length $l_{i}$ in each segment. The total length of the wire of the winding $L$ is given by the sum of contributions from each segment, $L=\sum_{i=0}^{i=N} n_{i} l_{i}$, and the average temperature is given by the expression

$$
\vartheta_{\mathrm{av}}=\frac{\sum_{i=0}^{i=N} n_{i} \int_{0}^{l_{i}} \vartheta_{i}(x) \mathrm{d} x}{\sum_{i=0}^{i=N} n_{i} l_{i}}
$$

This naturally leads to a definition of the average temperature for the segment $i$

$$
\vartheta_{\mathrm{av}, i}=\frac{1}{l_{i}} \int_{0}^{l_{i}} \vartheta_{i}(x) \mathrm{d} x
$$

Since the number of turns in each segment $n_{i}$ is proportional to the width of segments $h_{i}$, the average temperature is finally expressed as

$$
\vartheta_{\mathrm{av}}=\frac{\sum_{i=0}^{i=N} h_{i} l_{i} \vartheta_{\mathrm{av}, i}}{\sum_{i=0}^{i=N} h_{i} l_{i}}
$$


The temperature profile $\vartheta_{i}(x)$ of each segment fulfils an effective one-dimensional heat equation, used mostly for a description of cooling fins [6],

$$
\begin{aligned}
-\lambda_{\|} \frac{\mathrm{d}^{2} \vartheta_{i}(x)}{\mathrm{d} x^{2}}-\dot{q}+\frac{k_{i} \alpha_{i} \Gamma_{i}}{S_{i}}\left(\vartheta_{i}(x)-\vartheta_{\text {oil }}\right) & =0, \\
i & =0,1,2, \ldots N .
\end{aligned}
$$

Here, apart from the already introduced symbols, $\lambda_{\|}$is the thermal conductivity of the winding in the tangential direction, $\Gamma_{i}=2\left(h_{i}+l_{\mathrm{w}}\right)$ is the axial circumference of the segment and $S_{i}=h_{i} l_{\mathrm{w}}$ is the vertical cross-sectional area of the segment. $k_{i}$ is the ratio of the temperature rise on the surface of the winding $\Delta \vartheta_{\text {surface }}$ to the crosssectional average temperature rise in the winding $k=$ $\Delta \vartheta_{\text {surface }} / \Delta \vartheta$, both at a certain position $\mathrm{x}$ and defined as temperature rises above the average oil temperature. It is assumed that this ratio is independent of the position $x$. One can estimate its value from the result for the symmetric winding (1) as

$$
k=\left(1+\alpha h /\left(6 \lambda_{\perp}\right)\right)^{-1} .
$$

The $N+1$ differential equations (6) need to be supplemented by $2(N+1)$ boundary conditions. Due to the symmetry at $x_{i}=l_{i}$ we require

$$
\left.\frac{\mathrm{d} \vartheta_{i}}{\mathrm{~d} x}\right|_{l_{i}}=0, \quad i=0, \ldots, N
$$

The continuity of the temperature at $\mathrm{x}=0$ demands

$$
\vartheta_{0}(0)=\vartheta_{1}(0)=\cdots=\vartheta_{N}(0),
$$

which give further $N$ independent conditions. Finally, the heat flux conservation in the plane $O$ results in the last needed boundary condition

$$
\left.\sum_{i=0}^{N} S_{i} \lambda_{\|} \frac{\mathrm{d} \vartheta_{i}}{\mathrm{~d} x}\right|_{O}=0
$$

General solutions of the set of equations (6) and (8) are

$$
\vartheta_{i}(x)=\vartheta_{\text {oil }}+\gamma_{i}+B_{i} \cosh \left(\left(x-l_{i}\right) / \delta_{i}\right), \quad i=0, \ldots, N
$$

where

and

$$
\gamma_{i}=\frac{S_{i} \dot{q}}{k_{i} \alpha \Gamma_{i}}
$$

$$
\delta_{i}=\sqrt{\frac{\lambda_{\|} S_{i}}{\alpha k_{i} \Gamma_{i}}} .
$$

The coefficients $B_{i}$ are determined by the remaining conditions (9) and (10). We are interested in the average temperature of the segment (4) for which, using (11), we find

$$
\vartheta_{\mathrm{av}, i}=\vartheta_{\mathrm{oil}}+\gamma_{i}+\frac{B_{i} \delta_{i}}{l_{i}} \sinh \left(\frac{l_{i}}{\delta}\right) .
$$

Hence, instead of the coefficients $B_{i}$ it is convenient to introduce new coefficients $C_{i}=\left(B_{i} \delta_{i} / l_{i}\right) \sinh \left(l_{i} / \delta_{i}\right)$, for which the conditions (9) and (10) give

$$
\begin{aligned}
C_{i}=\frac{\delta_{i}}{l_{i}} \tanh \left(\frac{l_{i}}{\delta_{i}}\right)( & \gamma_{0}-\gamma_{i}- \\
& \left.\frac{\sum_{j=0}^{j=N} \frac{h_{j}}{\delta_{j}}\left(\gamma_{0}-\gamma_{j}\right) \tanh \left(\frac{l_{j}}{\delta_{j}}\right)}{\sum_{j=0}^{j=N} \frac{h_{j}}{\delta_{j}} \tanh \left(\frac{l_{j}}{\delta_{j}}\right)}\right) .
\end{aligned}
$$

In terms of these coefficients the average temperature of segment $i$ is simply

$$
\vartheta_{\mathrm{av}, i}=\vartheta_{\mathrm{oil}}+\gamma_{i}+C_{i}
$$

Equations (5), (13) and (14) are the main general result of this paper. They give a prescription for the calculation of the average temperature in a winding split by $N-2$ partial cooling ducts on both of its sides. However, the same results can be used also for $N-2$ partial cooling ducts on one side of the winding only, as can be easily seen from Fig. 3. The only difference is in the value one uses for the parameter $l_{0}$.

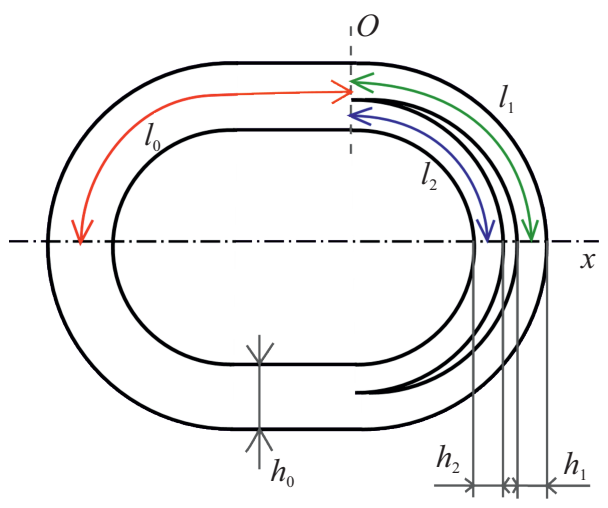

Fig. 3. Cross-sectional view of a winding with partial cooling duct on its right side only

The symmetry of this problem is weaker compared to the case in Fig. 2 but the mathematical model is the same. However, the ratio $\nu=l_{1} / l_{0}$ attains smaller value in this case.

Each contribution in (14) has a simple physical meaning - it corresponds to an additive term to the average temperature of the segment $i$. The second contribution $\gamma_{i}$ gives the temperature rise in a single segment if it were not connected to the other segments. In such a case the temperature within the segment along the tangential direction would be constant and the coefficients would be $C_{i}=0$. As one would expect, this contribution, in the regime $l_{w} \gg h_{i}$, is equal to the temperature rise in (1) for the corresponding value of $h_{i}$. The third contribution $C_{i}$ given by (13) is the correction resulting from the heat flow between different segments of the winding. 


\section{Simple but realistic example}

Let us now consider two simple but realistic examples of one cooling duct inserted into a winding on its single and both sides ie $(N=2)$. The latter corresponds to the situation in Fig. 2, the former to Fig. 3. We further simplify the situation by assuming that $l_{1}=l_{2} \equiv l_{\mathrm{d}}$ is the length and $h_{1}=h_{2} \equiv h_{\mathrm{d}}$ is the width of the segments with the duct. The width of the segment without the duct will be fixed to $h_{0}=2 h_{\mathrm{d}}$ which is a realistic choice. The length of this segment will be expressed through a ratio $\nu=l_{\mathrm{d}} / l_{0}$. According to (7) the factors $k_{i}$ are close to one so, for simplicity, we set them equal to one exactly. As a consequence of these assumptions one finds that the following also holds: $\gamma_{1}=\gamma_{2}=\gamma_{0} / 2 \equiv \gamma / 2, \delta_{1}=\delta_{2} \equiv \delta$ and $\delta_{0}=\sqrt{2} \delta$. Using these simplifications, one finds from (5), (13) and (14)

$$
\vartheta_{\mathrm{av}}=\vartheta_{\mathrm{oil}}+\frac{\gamma+C_{0}+\nu\left(\frac{\gamma}{2}+C_{1}\right)}{1+\nu}
$$

where

$$
\begin{gathered}
C_{0}=-\frac{\gamma \tanh \zeta}{2 Q \zeta}, \\
C_{1}=\frac{\gamma \tanh \xi}{2 \xi}\left(1-\frac{1}{Q}\right)
\end{gathered}
$$

and

$$
\xi=\frac{l_{\mathrm{d}}}{\delta}, \quad \zeta=\frac{l_{\mathrm{d}}}{\delta \nu \sqrt{2}}, \quad Q=1+\frac{\tanh \zeta}{\sqrt{2} \tanh \xi} .
$$

It is useful to cast these results into a nondimensional form. First, the length of the segment with the cooling duct $l_{\mathrm{d}}$ appears in (16-17) in the nondimensional ratio $\xi=l_{\mathrm{d}} / \delta$ only. Second, instead of the average temperature we introduce the relative temperature rise above the temperature of the oil,

$$
\Delta \vartheta_{\mathrm{av}, r}=\frac{\vartheta_{\mathrm{av}}-\vartheta_{\mathrm{oil}}}{\gamma}
$$

since all terms in (15-17) but $\vartheta_{\text {oil }}$ are proportional to $\gamma$. Hence, we calculate the temperature rise relative to the average temperature of the oil and in units of the temperature rise of a winding without the partial cooling duct $\gamma$.

The results (15-17) contain two dimensionless parameters: $\nu=l_{\mathrm{d}} / l_{0}$ and $\xi=l_{\mathrm{d}} / \delta$. For an oil-filled distribution transformer with nominal power $400 \mathrm{kVA}$ and a typical foil-type low voltage winding one typically finds $l_{\mathrm{d}} \approx 130 \mathrm{~mm}$.

For the design with cooling ducts at both sides on finds $l_{0} \sim 30 \mathrm{~mm}$ and for the design with one cooling duct $l_{0} \approx 200 \mathrm{~mm}$. All three lengths increase (decrease) with the increase (decrease) of the nominal power of the transformer, but their ratio remains roughly constant. Hence, we find two values of the ratio: $\nu=4.33$ for two and $\nu=0.65$ for a single partial cooling duct.
Roughly speaking, $\delta$ is the length on which the variation of the temperature along the circumference within a single segment relaxes to a constant value, as can be inferred from (11). The relaxation length $\delta$ can be estimated from (12) using the typical values used for the design of these transformers: $\lambda_{\|}=140 \mathrm{~W} / \mathrm{m} . \mathrm{K}$ (effective thermal conductivity along the tangential direction of the winding accounting for $\mathrm{Al}$ wire and the needed oil-soaked paper isolation), $S_{1} / \Gamma_{1}=h_{\mathrm{d}} l_{\mathrm{w}} /\left[2\left(h_{\mathrm{d}}+l_{\mathrm{w}}\right)\right] \approx$ $h_{\mathrm{d}} / 2=5 \mathrm{~mm}$ (for the oil-filled transformer mentioned above), $\alpha=80 \mathrm{~W} / \mathrm{m}^{2}$. K (for a typical mineral oil at the temperature $80[4,7])$ and $k_{1}=1$. Using these values, we obtain $\delta=100 \mathrm{~mm}$. From these considerations we obtain that for the $400 \mathrm{kVA}$ transformer $\lambda=l_{\mathrm{d}} / \delta \approx 1.3$ and hence a meaningful range for the value of the parameter for a variable nominal power might be from 0 to no more than 5 . The resulting dependence of the relative temperature rise as a function $\lambda=l_{\mathrm{d}} / \delta$ in this range, for the two above motivated values of the ratio $\nu$ is given in Fig. 4.

It is interesting to note that introducing a single partial cooling duct decreases the original temperature rise by almost $35 \%$. The additional duct on the other side of the winding adds to this decrease just another $15 \%$.

One can see that the described example is not very far from the zero limit of the functional form of the relative temperature rise indicated by the dashed lines in Fig. 4. The zero limit corresponds to a limit of very large thermal conductivity $\lambda_{\|}$. However, to quantify "how large", we need to check if $\xi=l_{\mathrm{d}} / \delta \ll 1$ is fulfilled. In this regime, starting from (14-16), one obtains a simple formula for the relative temperature rise

$$
\Delta \vartheta_{\mathrm{av}, r}^{0}=\frac{1+\nu}{1+2 \nu}
$$

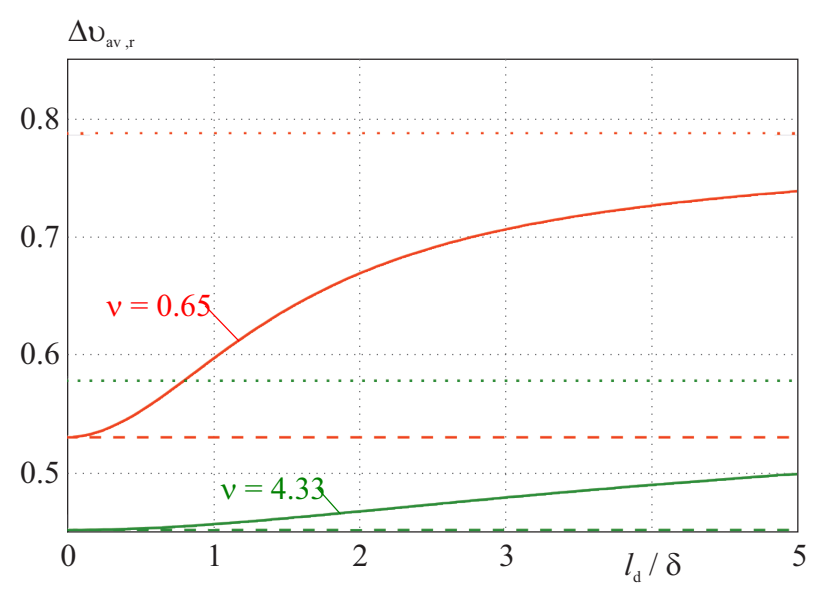

Fig. 4. Dependence of the relative temperature rise of a winding on the relative length of a partial cooling duct for one (red line, $\nu=0.65$ ) and two (green line, $\nu=4.33$ ) ducts in the design. The dotted and the dashed lines indicate the corresponding asymptotic values for zero and a very large value of the relaxation length $\delta$ for a given length of the cooling duct. 
For practical purposes this is a reasonable estimate of the magnitude of the decrease of temperature rise of a given winding modified by a partial cooling duct in one or both its sides. These two cases differ only by the value of the ratio $\nu=l_{\mathrm{d}} / l_{0}$. The result (18) has a simple interpretation - one can obtain the same expression assuming the temperature of all the segments are the same such, that via the Newton's law of heat transfer, all the dissipated Joule heat is passed into the oil through three parallel thermal resistances - one for the wider and two for the narrower segments. From this point of view, the presented derivations and example validate such a lump parameters model. This opens the possibility to account for partial cooling ducts using the popular approach based on an equivalent thermal circuit [8].

It is also interesting to analyse the limit of large $l_{\mathrm{d}} / \delta$, indicated in Fig. 4 with dotted lines. In this case the resulting limit gives

$$
\Delta \vartheta_{\mathrm{av}, r}^{\infty}=\frac{1+\nu / 2}{1+\nu}
$$

In contrast to (18), this result corresponds to "very small" values of the thermal conductivity $\lambda_{\|}$, ie $l_{\mathrm{d}} / \delta \gg 1$. Its lump parameter model would assume that each segment of the winding has its own temperature rise obtained from (1) and these are then averaged according to (5). This perhaps intuitively appealing approach is according to the here presented estimates worse than (18) but still can be used as the upper bound of the relative temperature rise.

\section{Conclusions}

A model has been presented that describes temperature variations in a winding with an arbitrary number of partial cooling ducts inserted into one or both of its sides. The average temperature has been defined in such a way as to correspond to the indirect measurement via the resistance of the winding. An analytical solution of the model has been presented. This general solution was then applied to a simple but realistic example of a low voltage winding of a $400 \mathrm{kVA}$ distribution transformer with one or two cooling ducts inserted. Starting from the exact solution a simple and practical formula for the temperature rise of such a winding has been derived that is suitable for a fast design of the windings. The validity of this formula has been discussed in terms of a dimensionless criterion.

\section{Acknowledgement}

The author would like to thank J. Lukáč for his comments on the history of the design of distribution transformers in BEZ Transformtory, a.s. This work was partly supported by the grant KEGA 002 STU-4/2019 "Heat transfer 2021".

\section{REFERENCES}

[1] E. I. Amoiralis, M. A. Tsili, and A. G. Kladas, "Transformer Design and Optimization: A Literature Survey", IEEE Transactions on power delivery, vol. 24, no. 4, pp. 1999-2024, 2009.

[2] "IEC Standard - Power Transformers: Temperature rise" Publication no. 76-2 1993.

[3] J. Lukáč, former head of the design department, BEZ Transformátory, a.s., private communication.

[4] E. Jezierski, Transformers, Bacic Theory (Transformátory, Teoretické základy), Praha: Academia, 1973, pp. 460-469 (in Czech).

[5] E. De Santis Junior, J. Mendes, A. P. Silva and S. Voss, (2016, May. 11) EP 3018667 A1, Accessed: January 20, 2021, https://worldwide.espacenet.com/patent/search?q=pn\%3DEP 3018667A1?\&section $=$ Biblio\&called_by $=\mathrm{EPAB}$.

[6] J. P. Holman, Heat transfer, 10 th ed. New York: McGraw-Hill, 2010 pp. $45-48$.

[7] K. Preis, O. Bíró,G. Buchgraber, and I. Tičar, "Thermal-Electromagnetic Coupling the Finite-Element Simulation of Power Transformers", IEEE Transactions on magnetics, vol. 42, no. 4, pp. 999-1002, 2006.

[8] G. Swift, T. S. Molinski, and W. Lehn, "A Fundamental Approach to Transformer Thermal Modeling, Part I: Theory and Equivalent Circuit", IEEE Transactions on power delivery, vol. 16, no. 2, pp. 171-175, 2001.

Received 27 December 2020

Peter Bokes (Doc, Dr, Ing), born in 1973, graduated from the Tokyo Institute of Technology in 1998, and obtained his $\mathrm{PhD}$ degree at the Slovak University of Technology in Bratislava, Faculty of Electrical Engineering and Information Technology (FEI STU) in 2001. At present he is Associated Professor at the Institute of Nuclear and Physical Engineering, FEI STU Bratislava. As a postdoctoral researcher he worked at the University of York during the years 2001-2003 and 2007-2008. In the period 2014-2016 he worked as the head of research at BEZ Transformátory, a.s. His research interest span from quantum mechanics and electronic structure theory and simulations to FEM simulations of heat transfer and transformers design. 